\title{
Survival and prognostic factors of lung cancer patients with preexisting connective tissue disease: a retrospective cohort study
}

\author{
Huaxia Yang ${ }^{1 \#}$, Zhuoran Yao ${ }^{1 \#}$, Xiaoxiang Zhou ${ }^{1 \#}$, Zhongxing Bing ${ }^{2}$, Lei $\mathrm{Cao}^{2}$, Zhili $\mathrm{Cao}^{2}$, Shanqing $\mathrm{Li}^{2}$, \\ Xuan Zhang ${ }^{1}$, Yan Zhao ${ }^{1}$, Xiaofeng Zeng ${ }^{1}$, Fengchun Zhang ${ }^{1}$, Naixin Liang ${ }^{2}$ \\ ${ }^{1}$ Department of Rheumatology and Clinical Immunology, Peking Union Medical College Hospital, Chinese Academy of Medical Science \& Peking \\ Union Medical College, Key Laboratory of Rheumatology and Clinical Immunology, National Clinical Research Center for Dermatologic and \\ Immunologic Diseases, Beijing, China; ${ }^{2}$ Department of Thoracic surgery, Peking Union Medical College Hospital, Chinese Academy of Medical \\ Science \& Peking Union Medical College, Beijing, China \\ Contributions: (I) Conception and design: H Yang, Z Yao, X Zhou, N Liang; (II) Administrative support: X Zhang, X Zeng, F Zhang; (III) Provision of \\ study materials or patients: N Liang; (IV) Collection and assembly of data: H Yang, Z Yao, X Zhou; (V) Data analysis and interpretation: All authors; \\ (VI) Manuscript writing: All authors; (VII) Final approval of manuscript: All authors. \\ "These authors contributed equally to this work. \\ Correspondence to: Naixin Liang, MD. Department of Thoracic surgery, Peking Union Medical College Hospital, Chinese Academy of Medical \\ Science, Beijing 100730, China. Email: pumchnelson@163.com.
}

Background: Connective tissue diseases (CTDs) are a group of special commodities in lung cancer (LC). This study aimed to analyze the survival and prognostic factors of LC patients with preexisting CTDs.

Methods: A total of $84 \mathrm{LC}$ patients with preexisting CTDs that presented at Peking Union Medical College Hospital (PUMCH) were retrospectively recruited in this study between January 2000 and June 2017. Patient survival was compared using the Kaplan-Meier method. Univariate and multivariate Cox proportional hazard models were used to assess prognostic variables.

Results: Of the 84 LC patients, 36 (41.8\%) had underlying rheumatoid arthritis (RA), 20 (23.8\%) had idiopathic inflammatory myopathy (IIM), 18 (21.4\%) had Sjögren syndrome (SS), 6 (7.1\%) had systemic sclerosis (SSc), and 4 (4.8\%) had systemic lupus erythematosus (SLE). The median overall survival (OS) was 21 months (IQR, 8-72 months), and the 1-, 3-, and 5-year survival rates were $61.3 \%, 36.7 \%$, and $29.5 \%$, respectively. The survival rates between different CTD subgroups, histopathologies, and disease stages were significantly different $(\mathrm{P}<0.05)$. Multivariate analysis showed that the independent prognostic factors for OS were IIM [hazard ratio (HR), 3.61; 95\% confidence intervals (CI), 1.69-8.21; P=0.002], SS (HR, 2.72; 95\% CI, 1.01-7.33; $\mathrm{P}=0.048$ ), and radical resection ( $\mathrm{HR}, 0.11 ; 95 \% \mathrm{CI}, 0.04-0.35 ; \mathrm{P}<0.001$ ).

Conclusions: Different CTD subtypes and the radical resection of LC are closely related to patient prognosis. This indicates a need for both identifications of CTD types and active treatment strategies for LC.

Keywords: Lung cancer; connective tissue disease (CTDs); survival; prognostic factors

Submitted Jan 28, 2020. Accepted for publication Aug 19, 2020.

doi: 10.21037/atm-20-1072

View this article at: http://dx.doi.org/10.21037/atm-20-1072

\section{Introduction}

Connective tissue diseases (CTDs) are associated with a variety of malignancies, one such malignancy being lung cancer (LC), which attracts major attention due to its high incidence and mortality rate (1). Approximately $14-25 \%$ of LC patients are reported also to have rheumatic diseases $(2,3)$. Similarly, individuals with underlying CTDs, including systemic lupus erythematosus (SLE), rheumatoid 
arthritis (RA), idiopathic inflammatory myopathy (IIM), Sjögren syndrome (SS), and systemic sclerosis (SSc) have an increased risk of LC (4-6).

Although a great deal of evidence supports the notion of a close association between CTDs and LC, when it comes to the prognosis of LC patients with preexisting CTDs, data is scarce and frequently conflicting. A retrospective cohort study reported that LC patients with RA and IIM might have worse survival outcomes than those without CTDs by presenting an advanced stage, more comorbidities and poorer functional status (7). However, a recent study found no significant difference in outcomes between LC patients with and without underlying RA, suggesting that certain types of CTDs may not negatively influence LC prognosis (8).

When an LC patient has preexisting CTDs, it is challenging for clinicians to determine the most suitable treatment. Oncologists tend to be conservative when managing systemic autoimmune diseases such as SLE, RA, and SSc. As a result, patients with CTDs are universally excluded from clinical trials or even standard therapeutics. However, evidence concerning the outcomes of patients with LC and preexisting CTDs that received different treatments is still lacking.

In this retrospective cohort study, we identified a wellcharacterized population of LC patients with preexisting CTDs including SLE, RA, IIM, SS, and SSc and performed long-term follow-up. We examined the clinical characteristics and survival rates of this cohort and evaluated the associations between baseline patient features (CTD subtype, LC staging, pathological type, and treatment) and survival outcomes of LC patients with preexisting CTDs. This study was carried out following the STROBE checklist (available at http://dx.doi.org/10.21037/atm-20-1072).

\section{Methods}

\section{Study population}

We retrospectively reviewed the medical records of 13,871 LC patients diagnosed between January 2000 and June 2017 (Figure 1). All LC patients with preexisting CTDs were consecutively enrolled in this study and followed up at the Department of Thoracic Surgery and the Department of Rheumatology and Clinical Immunology, Peking Union Medical College Hospital (PUMCH). We adopted the following inclusion criteria:

* Patients were histopathologically diagnosed with LC by biopsy or resected surgical specimens according to the International Classification of Disease 10 (ICD-10) criteria. Histological groups were defined based on the International Classification of Disease for Oncology Version 3 (ICD-O-3) morphology codes and categorized as adenocarcinoma, squamous cell carcinoma, or neuroendocrine carcinoma. LCs were retrospectively staged based on patient surgicalpathological records, according to the American Joint Committee on Cancer (AJCC) Staging Manual $7^{\text {th }}$ edition.

* Patients with preexisting CTDs diagnosed at any time before their LC diagnosis, including RA, IIM, SS, SSc, and SLE. RA, SLE, SS, SSc, and IIM were retrieved according to the 1987 revised classification criteria of the American College of Rheumatology (ACR) for RA, the 1997 ACR classification criteria for SLE, the 2002 American-European Consensus Group criteria for SS, the 1980 preliminary ACR classification criteria for SSc, and the Bohan and Peter criteria for IIM, respectively (9-13).

Patients that did not meet the inclusion criteria were excluded. Other exclusion criteria included the following:

* Patients diagnosed with CTDs following their LC diagnosis.

* Patients with overlapping rheumatic diseases.

This study was approved by the Medical Ethics Committee of PUMCH and complied with all ethics committee requirements (protocol ID: S-K1019). Written informed consents have been obtained from all participants. And the study was conducted in accordance with the Declaration of Helsinki (as revised in 2013).

\section{Data collection}

We obtained patient data, including baseline demographics, personal histories, clinical manifestations of LC and CTD, treatment, and follow-up from their medical records. Personal histories included smoking, a family history of malignancies, and a family history of LC. Duration of LC and preexisting CTDs were calculated from the initial time of diagnosis of CTDs to the diagnosis of LC. The patients' baseline staging and histological diagnoses were acquired, and the LC treatment they received, including surgical resection, chemotherapy, targeted therapy, and radiotherapy, was recorded. The patients were followed up, and each patient's prognosis was documented. The patients' survival time was the interval between the first pathologic 


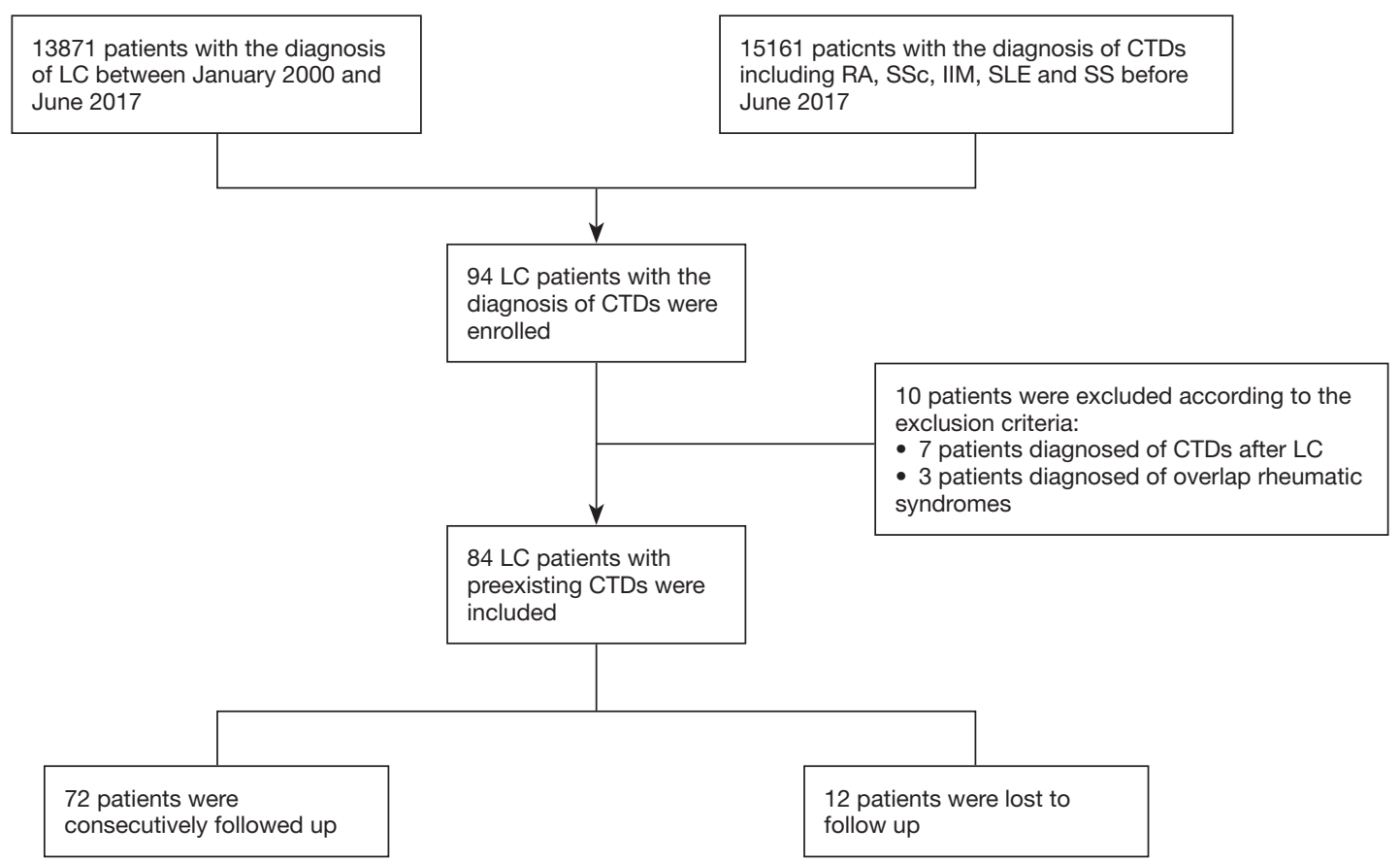

Figure 1 Flow chart of inclusion criteria, exclusion criteria, and follow-up of LC patients with preexisting CTDs. CTD, connective tissue disease; LC, lung cancer; IIM, idiopathic inflammatory myopathy; RA, rheumatoid arthritis; SS, Sjögren syndrome; SSc, systemic sclerosis; SLE, systemic lupus erythematosus.

diagnosis of LC and either the recorded date of death or 1 September 2019 (the censoring date).

\section{Statistical analysis}

Continuous variables were summarized as either mean \pm standard deviation (SD) or median and interquartile range (IQR), while categorical data were presented as frequencies (percentages). Analysis of variance (ANOVA) and chi-squared tests for independence was used to evaluate significant differences in continuous data and categorical data between groups, respectively. Follow-up began at the time of cancer diagnosis and was censored either at the time of death or on the last day of survival status follow-up, whichever came first. Survival curves were generated using the Kaplan-Meier method. Differences in survival were compared using the log-rank test. The Cox proportional hazards model was used to assess the associations between clinical variables and survival. Only variables found to have a $\mathrm{P}$ value of 0.1 or less in the univariate analysis were included in the multivariate model. All statistical analyses were performed with SPSS software (version 23.0), and all survival data were analyzed using GraphPad Prism (version
6.0). $\mathrm{P}$ values of less than 0.05 were considered statistically significant.

\section{Results}

\section{Demographics and clinical characteristics of $L C$ patients with preexisting CTDs}

A total of 13,871 patients diagnosed with LC between January 2000 and June 2017 were identified. Of these patients, 94 also had CTDs, including SLE, SS, RA, IIM, and SSc. Of these 94 patients, 7 were excluded due to their CTD diagnosis following their LC diagnosis, and 3 were excluded due to overlapping rheumatic diseases. A total of 84 LC patients with preexisting CTDs were therefore enrolled in this study, including $36(41.8 \%)$ with RA, 20 $(23.8 \%)$ with IIM, 18 (21.4\%) with SS, 6 (7.1\%) with SSc, and $4(4.8 \%)$ with SLE (Table 1 and Figure 1). Of these patients, $37(44.0 \%)$ were male and $47(56.0 \%)$ were female. The mean age was $52.3 \pm 14$ years at the time of preexisting CTD diagnosis and $60.5 \pm 10.3$ years at the time of LC diagnosis. The median time interval between CTD and LC diagnosis was 64 months (IQR, 8-162 months) (Table 1). LC was diagnosed a median of 4 months (IQR, 
Table 1 Demographic and clinical features of 84 patients with LC and CTDs

\begin{tabular}{|c|c|c|c|c|c|c|c|}
\hline Patients & $\begin{array}{l}\text { All CTDs } \\
(\mathrm{n}=84)\end{array}$ & $\mathrm{RA}(\mathrm{n}=36)$ & IIM $(n=20)$ & $S S(n=18)$ & $\operatorname{SSc}(n=6)$ & SLE $(n=4)$ & $P$ value \\
\hline \multicolumn{8}{|l|}{ Demographic features } \\
\hline Age at CTD, dx, years & $52.3 \pm 14.0$ & $52.1 \pm 13.1$ & $62.0 \pm 10.6$ & $50.8 \pm 12.3$ & $31.2 \pm 5.6$ & $43.0 \pm 12.4$ & $<0.001$ \\
\hline \multicolumn{8}{|l|}{ Personal history } \\
\hline Smoking history & $39(46.4)$ & $17(47.2)$ & $16(80.0)$ & $4(22.2)$ & $0(0.0)$ & $2(50.0)$ & 0.001 \\
\hline Family history of all malignancies & $17(20.2)$ & $2(5.6)$ & $4(20)$ & 7 (38.9) & $3(50.0)$ & $1(25.0)$ & 0.017 \\
\hline Epithelial tumors & $66(78.6)$ & $29(80.6)$ & $14(70.0)$ & $15(83.3)$ & $5(83.3)$ & $3(75.0)$ & 0.860 \\
\hline Adenocarcinoma & $52(61.9)$ & $20(55.6)$ & $10(50.0)$ & $15(83.3)$ & $4(66.7)$ & $3(75.0)$ & 0.225 \\
\hline Squamous cell carcinoma & $14(16.7)$ & $9(25.0)$ & $4(20.0)$ & $0(0.0)$ & $1(16.7)$ & $0(0.0)$ & 0.174 \\
\hline Neuroendocrine carcinoma & $18(21.4)$ & $7(19.4)$ & $6(30.0)$ & $3(16.7)$ & $1(16.7)$ & $1(25.0)$ & 0.860 \\
\hline Small cell carcinoma & $13(15.5)$ & $4(11.1)$ & $5(25.0)$ & $3(16.7)$ & $1(16.7)$ & $0(0.0)$ & 0.736 \\
\hline Others $^{a}$ & $5(6.0)$ & $3(8.3)$ & $1(5.0)$ & $0(0.0)$ & $0(0.0)$ & $1(25.0)$ & 0.342 \\
\hline \multicolumn{8}{|l|}{ Surgical-pathological stage } \\
\hline Early stage & $29(34.5)$ & $16(44.4)$ & $5(25.0)$ & $6(33.3)$ & $2(33.3)$ & $0(0.0)$ & \\
\hline Late stage & $55(65.5)$ & $20(55.6)$ & $15(75.0)$ & $12(66.7)$ & $4(66.7)$ & $4(100.0)$ & 0.343 \\
\hline \multicolumn{8}{|l|}{ Treatment } \\
\hline Radical resection & $30(35.7)$ & $16(44.4)$ & $6(30.0)$ & $6(33.3)$ & $2(33.3)$ & $0(0.0)$ & 0.439 \\
\hline Chemotherapy & $47(56.0)$ & $22(61.1)$ & $11(55.0)$ & $7(38.9)$ & $4(66.7)$ & $3(75.0)$ & 0.495 \\
\hline Target therapy & $25(29.8)$ & $10(27.8)$ & $3(15.0)$ & $8(44.4)$ & $3(50.0)$ & $1(25.0)$ & 0.265 \\
\hline Radiotherapy & $20(23.8)$ & $6(16.7)$ & $8(40)$ & $4(22.2)$ & $2(33.3)$ & $0(0.0)$ & 0.242 \\
\hline \multicolumn{8}{|l|}{ Follow-up ${ }^{c}$} \\
\hline Follow-up period, months & 65 [25-98] & 67 [26-104] & 67 [25-102] & 54 [27-79] & 65 [31-110] & 39 [2-102] & 0.879 \\
\hline OS, months & $21[8-72]$ & $34[18-72]$ & 9 [4-31] & 20 [6-72] & 53 [11-NE] & $7[5-14]$ & 0.021 \\
\hline
\end{tabular}

Data are expressed as mean \pm SD, median $[\mathrm{IQR}]$ or as percentage (\%). ${ }^{a}$, thers includes large cell neuroendocrine carcinoma and carcinoid. ${ }^{b}$, early stage is defined as stage I-IIla LC and late stage is defined as stage IIlb to IV LC. ' , the data was available for 72 patients. CTD, connective tissue disease; RA, rheumatoid arthritis; IIM, idiopathic inflammatory myopathy; SS, Sjögren syndrome; SSc, systemic sclerosis; SLE, systemic lupus erythematosus; Dx, diagnosis; LC, lung cancer; OS, overall survival; NE, not evaluable. 
2-8 months) after IIM diagnosis, while the time interval between RA, SS, SSc, and SLE diagnosis and LC diagnosis ranged from 0 to over 30 years (Table 1 and Figure 2). Of these 84 enrolled patients, epithelial tumors were identified in 66 patients $(78.6 \%)$, which included $52(61.9 \%)$ with adenocarcinoma and $14(16.7 \%)$ with squamous cell carcinoma. Neuroendocrine carcinomas were present in 18 patients $(21.4 \%)$, while $13(15.5 \%)$ had small cell carcinomas. According to the AJCC, 16 (19.0\%) patients presented with stage I disease, $13(15.5 \%)$ with stage II, 5 (6.0\%) with stage III, and 50 (59.5\%) with stage IV.

Gender distribution, the patient's age at CTD diagnosis, the interval between the patient's preexisting CTD diagnosis and LC diagnosis, and personal history, including smoking and familial malignancy, were significantly different among the five major preexisting CTD subgroups $(\mathrm{P}<0.05)$. Patients with IIM, in particular, showed enormous heterogeneity. LC patients with IIM included a higher proportion of male patients and smokers. Additionally, the length of time between IIM and LC diagnoses was considerably shorter compared with RA, SLE, SS, and SSc (Figure 2). Other clinical variables, including a family history of LC, histopathology, cancer stage, and LC treatment, showed no significant differences among the subgroups $(\mathrm{P}>0.05)$.

\section{Treatment and survival of LC patients with preexisting CTDs}

During the median follow-up of 65 months (IQR, 2598 months), 30 patients (35.7\%) received radical resections, whereas $47(56.0 \%)$ had chemotherapy, 25 (29.8\%) had targeted therapy, and 20 (23.8\%) had radiotherapy (Table 1). 12 patients were lost to follow up. The median overall survival (OS) was 21 months (IQR, 8-72 months), and the 1 -, 3 -, and 5 -year survival rates were $61.3 \%, 36.7 \%$, and $29.5 \%$, respectively (Figure $3 A$ ). A total of 50 deaths (50/72; $69.4 \%$ ) were reported, with malignancy being the leading cause of death.

Kaplan-Meier survival curves were drawn based on different CTD subtypes (Figure 3B), tumor, node, metastasis (TNM) stages (Figure 3C), and LC histopathology (Figure $3 D, E$ ). Survival rates were significantly different between the CTD subtypes $(\mathrm{P}=0.021)$. Patients with tumors of an epithelial origin had a better prognosis than those with neuroendocrine carcinoma (a median survival time of 31 months compared with 11 months; $\mathrm{P}=0.039$ ). No

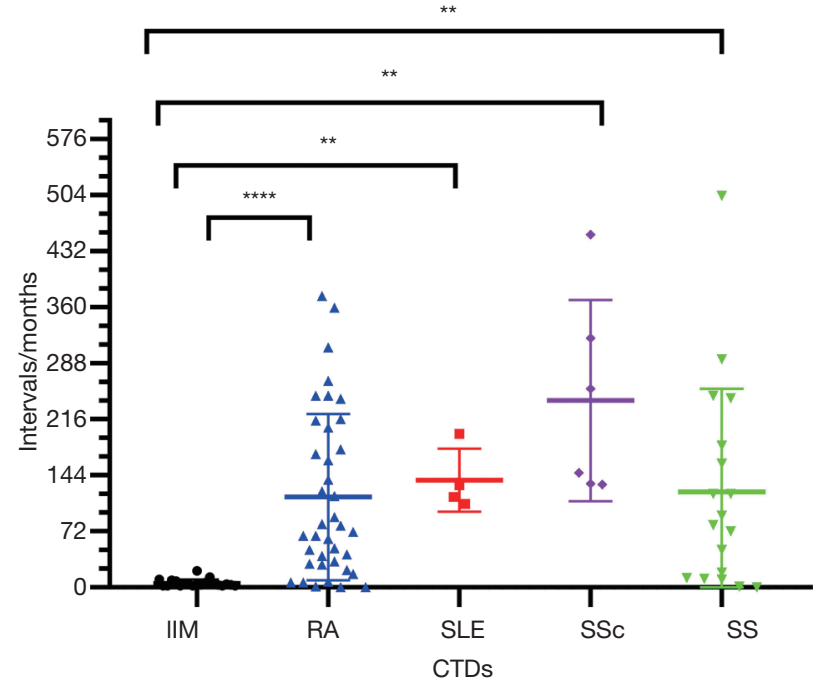

Figure 2 Comparisons of LC-CTD intervals between the five CTD groups. CTD, connective tissue disease. LC, lung cancer; IIM, idiopathic inflammatory myopathy; RA, rheumatoid arthritis; SS, Sjögren syndrome; SSc, systemic sclerosis; SLE, systemic lupus erythematosus. ${ }^{* *} \mathrm{P}<0.01 ;{ }^{* * * *} \mathrm{P}<0.0001$.

difference was observed between patients with squamous cell carcinoma and those with adenocarcinoma. There was a significant difference in prognosis between patients at different LC stages $(\mathrm{P}<0.001)$. Additionally, the stagespecific prognoses of patients receiving different treatments were recorded and analyzed (Figure 4). Notably, for early stage LC (defined as stage I-IIIa by the AJCC staging system), patients with preexisting CTDs that received radical resections demonstrated significantly longer survival than patients who did not receive radical resections (a median survival time not reached compared with 6 months; $\mathrm{P}=0.001$ ) (Figure 4A). Regarding of late stage $\mathrm{LC}$, including stage IIIb to IV lung cancers, a difference in overall survival of chemotherapy, targeted therapy as well as radiotherapy compared with their counterpart could not be identified $(\mathrm{P}>0.05)$ (Figure 4B-D).

\section{Prognostic analysis for LC patients with preexisting CTDs}

The results of the univariate analyses of LC patients with preexisting CTDs are summarized in Table 2. The prognostic factors selected from the univariate analysis for use in the multivariate analysis were as follows: smoking history (HR, 2.12; 95\% CI, 1.20-3.72; $\mathrm{P}=0.009$ ), IIM (HR, 2.62; 95\% CI, 1.29-5.32; $\mathrm{P}=0.008)$, SLE (HR, 5.07; 95\% 

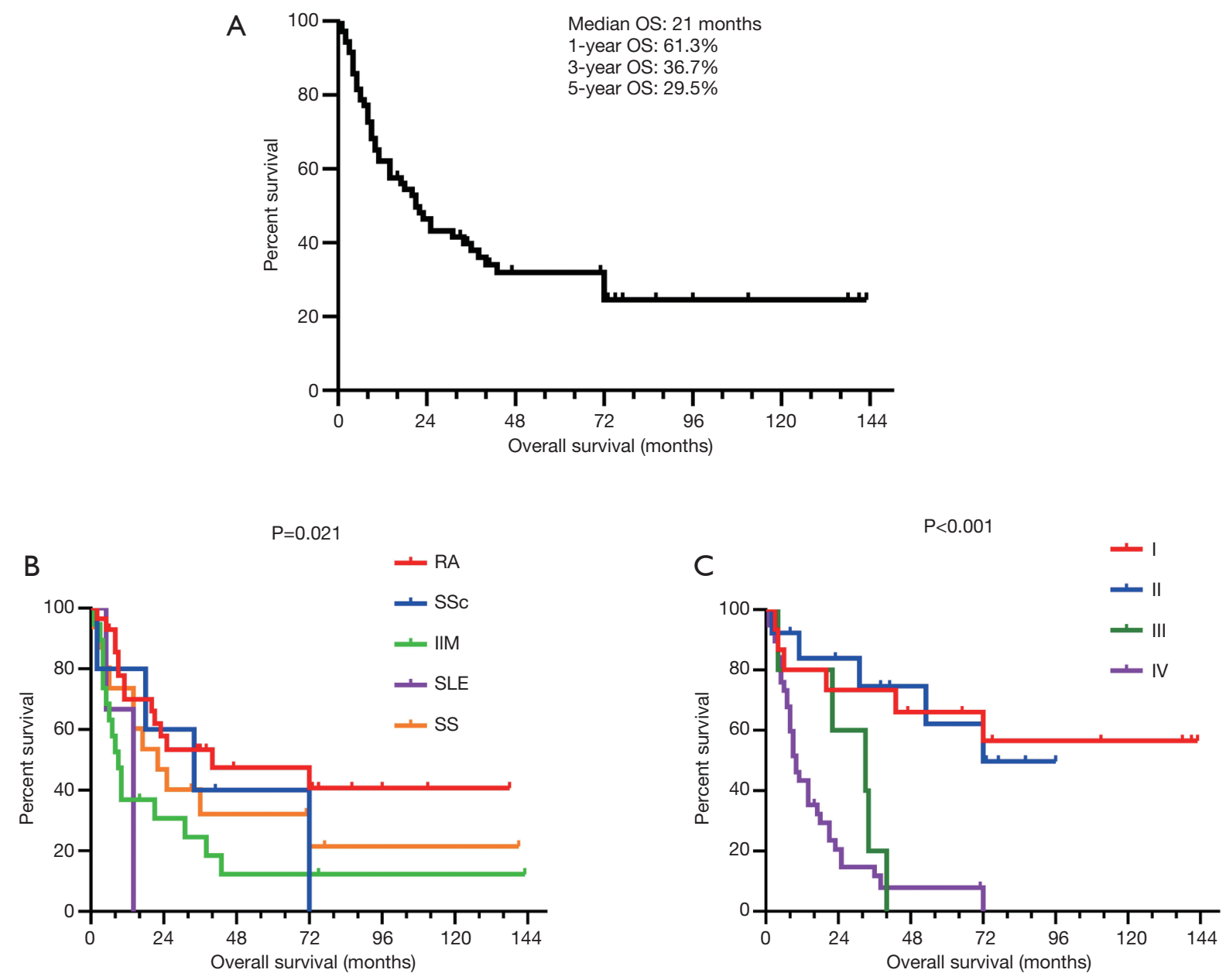

D

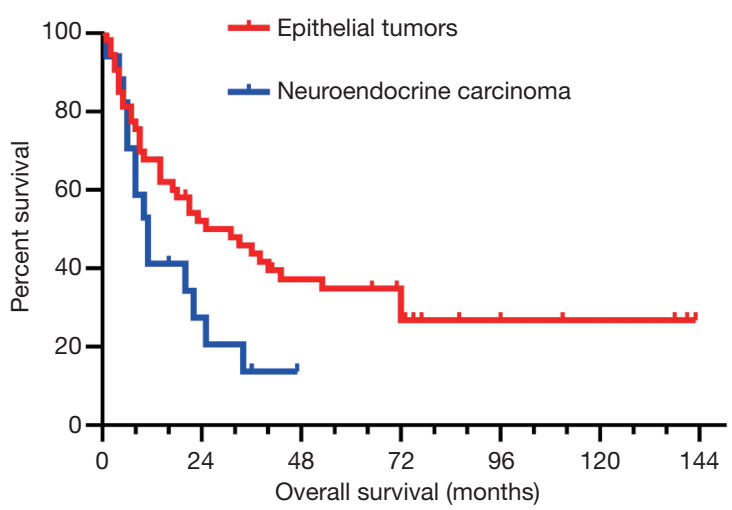

$\mathrm{E}$

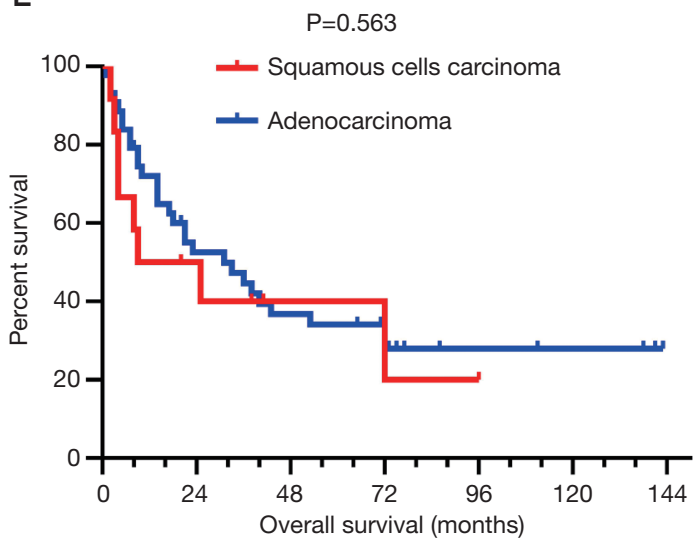

Figure 3 Survival curves. (A) Survival curve for LC patients with all CTDs; (B) survival curves for LC patients according to the five CTD subgroups; (C) survival curves for LC patients according to surgical-pathological stages; (D) survival curves for LC patients according to the two major histopathological subgroups; (E) comparison of survival between epithelial tumors. LC, lung cancer; CTD, connective tissue disease; OS, overall survival; IIM, idiopathic inflammatory myopathy; RA, rheumatoid arthritis; SS, Sjögren syndrome; SSc, systemic sclerosis; SLE, systemic lupus erythematosus. 
A

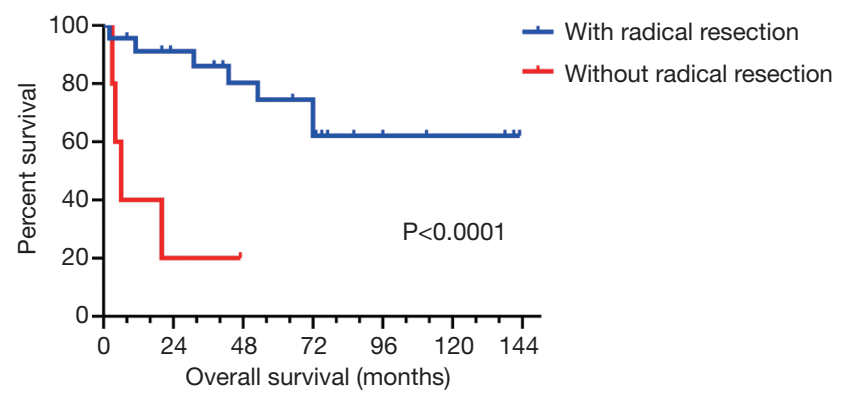

C

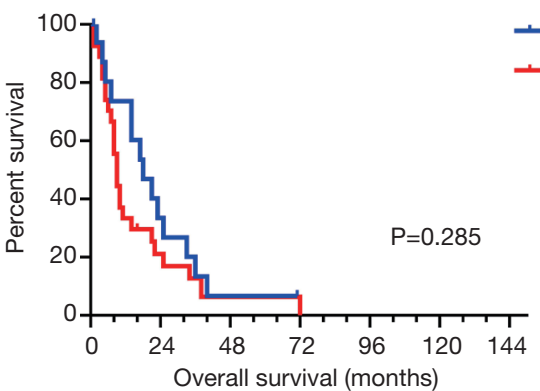

B

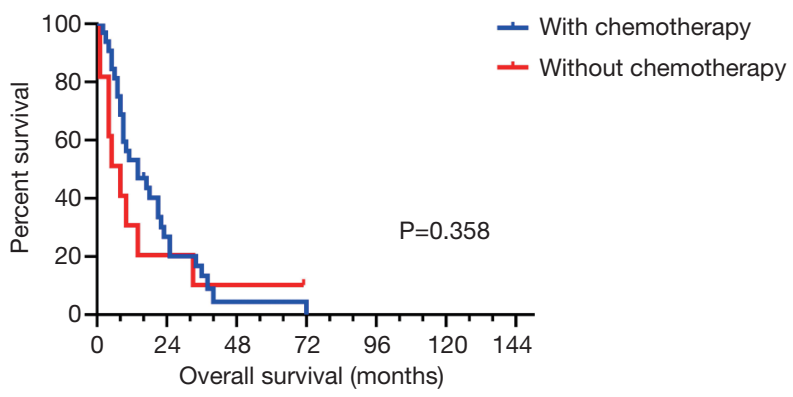

D

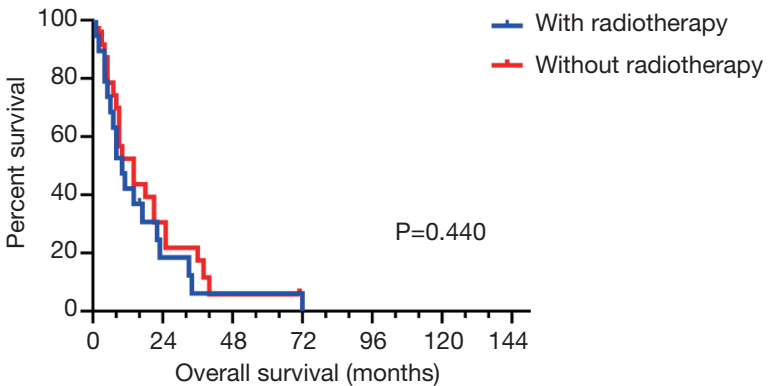

Figure 4 Survival curves of different treatments. (A) Comparison of survival curves for early stage LC-CTD patients with/without radical resection. (B) Comparison of survival curves for late-stage LC-CTD patients with/without chemotherapy. (C) Comparison of survival curves for late-stage LC-CTD patients with/without targeted therapy. (D) Comparison of survival curves for late-stage LC-CTD patients with/ without radiotherapy. LC, lung cancer; CTD, connective tissue disease; IIM, idiopathic inflammatory myopathy; RA, rheumatoid arthritis; SS, Sjögren syndrome; SSc, systemic sclerosis; SLE, systemic lupus erythematosus. The early stage is defined as stage I-IIIa LC. Late-stage is defined as stage IIIb to IV LC.

CI, 1.42-18.18; $\mathrm{P}=0.013)$, neuroendocrine carcinoma (HR, 1.92; 95\% CI, 1.02-3.64; $\mathrm{P}=0.045)$, late stage LC (HR, 5.77; 95\% CI, 2.79-11.94; $\mathrm{P}<0.001)$, and radical resection (HR, 0.12; 95\% CI, 0.059-0.251; $\mathrm{P}<0.001$ ) (Table 2). The multivariate analysis summarized in Table 3 indicates that IIM (HR 3.61; 95\% CI, 1.69-8.21; P=0.002) and SS (HR, 2.72; 95\% CI, 1.01-7.33; $\mathrm{P}=0.048$ ) were both independently associated with worse OS. Radical resection (HR, 0.11; 95\% CI, 0.04-0.35; $\mathrm{P}<0.001$ ) was found to be an independent prognostic factor for longer survival (Table 3).

\section{Discussion}

We examined the prognosis of patients with LC and preexisting CTDs and evaluated the potential associations between clinical characteristics and survival using a relatively large retrospective cohort with a long-term follow-up period. Given the rarity of LC and CTDs as comorbidities, it is unsurprising that most previous studies focused on comparing cohorts of CTD patients with non-CTD controls in the general LC population. To our knowledge, this is the first prognostic analysis of patients with LC and CTDs of such a large sample size that involved such detailed baseline characteristics and follow-up, and this is the first study to provide evidence of preferable treatment options for this particular patient group.

Although all CTDs arise due to a defective immune response with subsequent chronic inflammation, the difference between CTDs in immune dysregulation can lead to a variety of clinical manifestations on development of malignancy. IIM was shown to be markedly different from other CTDs: the age of IIM diagnosis was older than for other CTD subgroups, and it had a higher male to female ratio and more smokers, which was consistent with a series of previous studies that indicated an older age at diagnosis and the male sex are the demographic parameters most commonly associated with an increased risk of malignancy in IIM patients (14-17). Notably, the 
Table 2 Univariate analysis for overall survival of patients with LC and CTDs

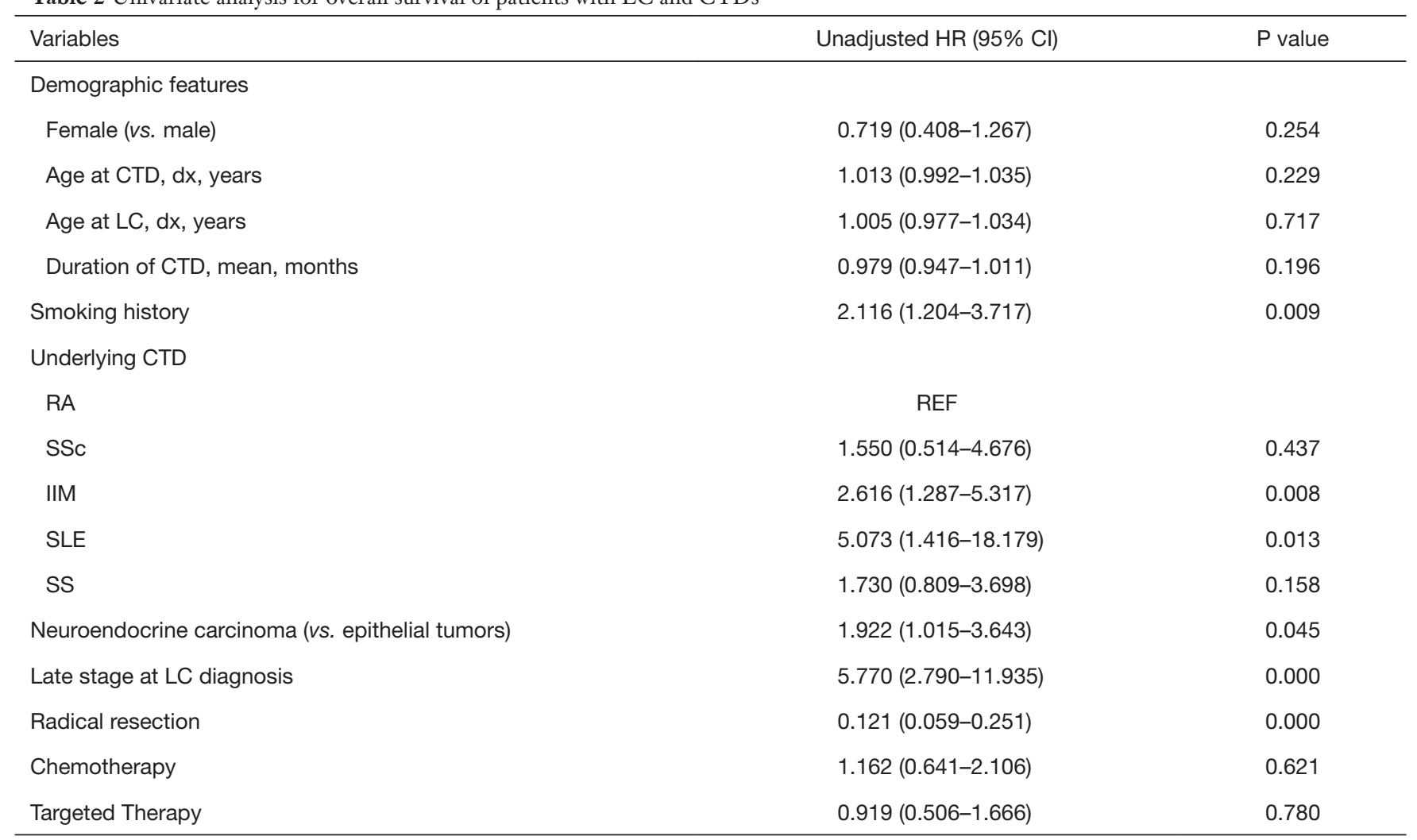

LC, lung cancer; CTD, connective tissue disease; HR, hazard ratio; Dx, diagnosis; REF, reference; RA, rheumatoid arthritis; SSc, systemic sclerosis; IIM, idiopathic inflammatory myopathy; SLE, systemic lupus erythematosus; SS, Sjögren syndrome.

time interval between IIM and LC diagnoses was no longer than a year, in contrast with the time interval between the onset of other CTDs and LC, which appears to be sporadic and can take longer than 10 years. Similarly, several studies have found that the risk of malignancy is highest in the first year after IIM diagnosis $(18,19)$.

Considering that most autoimmune diseases are associated with the female sex and young age, the distinctive demographic features of LC patients with preexisting IIM indicate underlying pathogenesis unique from other CTDs. While the consequences of chronic inflammation, such as increased production of inflammatory cytokines and reactive oxygen species, mitochondrial damage, DNA methylation, and other changes in cellular metabolism and signaling, are possibly attributed to cancer progression in CTD patients, several lines of evidence support the hypothesis that a cancer-triggered autoimmune mechanism is involved in IIM pathogenesis (20-23). Self-protein mutations or overexpression of autoantigen in tumors could initiate an immune response that is cross-reactive with healthy tissues (24). In our study, most LC cases were diagnosed while patients were hospitalized for myositis, suggesting that LC may progress earlier than the onset of cutaneous-vascular manifestations.

The 5-year OS for the cohort of patients with LC and preexisting CTDs was $29.5 \%$, and the median OS duration was 21 months. These results were inconsistent with that of previous studies due to differences in cancer stage distribution (25). The univariate analysis showed that patients with a smoking history, a later cancer stage, neuroendocrine carcinoma, and the CTD subtypes of SLE and IIM had significantly worse OS, whereas patients that received radical resection appeared to have longer survival. Nevertheless, these prognostic factors are closely related, which can result in misinterpretation of their prognostic value. We, therefore, conducted a subsequent multivariate analysis to identify independent prognostic factors for cohorts with LC and preexisting CTDs. After adjustment, IIM and SS were revealed to be correlated with worse survival outcomes, and radical resection was still found to 
Table 3 Multivariate analysis for overall survival of patients with LC and CTDs

\begin{tabular}{lcc}
\hline Variables & Adjusted HR (95\% Cl) & P value \\
\hline Smoking history & $1.284(0.549-3.001)$ & 0.564 \\
Underlying CTD & REF & \\
RA & $1.977(0.546-7.152)$ & 0.299 \\
SSC & $3.609(1.586-8.209)$ & 0.002 \\
IIM & $3.201(0.861-11.930)$ & 0.083 \\
SLE & $2.720(1.010-7.330)$ & 0.048 \\
SS & $1.359(0.501-3.687)$ & 0.547 \\
Late stage at LC diagnosis & $1.271(0.628-2.573)$ & 0.505 \\
Neuroendocrine carcinoma & $0.111(0.036-0.349)$ & 0.000 \\
Radical resection & &
\end{tabular}

LC, lung cancer; CTD, connective tissue disease; HR, hazard ratio; REF, reference; RA, rheumatoid arthritis; SSc, systemic sclerosis; IIM, idiopathic inflammatory myopathy; SLE, systemic lupus erythematosus; SS, Sjögren syndrome.

play a protective role. Using RA as a reference, IIM and SS patients had significantly higher mortality. SLE patients also showed a trend of worse survival. However, this trend was not statistically significant. The distinct outcomes between CTD subgroups suggest they may have diverse impacts on LC biology; the differences between immune dysregulation and corresponding immunosuppressants in these subgroups might influence survival outcomes. Of note is that our findings contradicted a previous report that RA negatively affects the outcomes of LC patients compared with SSc patients, which indicates a need for studies of larger sample sizes in the future (7).

Comorbidities are always challenging for oncologists. Our study provided some preliminary evidence on therapeutic modalities for patients with LC and preexisting CTDs. As LC treatment varies greatly depending on its stage, we drew separate survival curves in a stage-specific manner. Radical resection markedly improved OS in early stage LC patients, which is consistent with our multivariate analysis. Patients with late-stage LCs, which were mostly inoperable, tended to show minor improvements in OS when they received chemotherapy and targeted therapy, although this therapeutic benefit was not statistically significant. This suggests that LC patients with CTDs should be treated with comparable aggressiveness to those without CTDs if partial control of the primary disease can be achieved. Currently, with the rapid evolution of cancer immunotherapy, CTDs would be increasingly addressed under the massive application of immune checkpoint inhibitors.

Several studies have addressed the prognosis of patients with LC and preexisting CTDs (Table S1). Most previous studies focused on comparing cancer survival rates between patients with and without CTDs (8,25-29). In 2008, Adzić et al. reported on the clinical features of 24 patients with LC and CTDs. While the baseline characteristics were documented in detail, due to the limited sample size, a prognosis analysis could not be conducted (25). Nayak et al. analyzed the impact of RA on the mortality of cancer patients using a large-scale cohort. However, this study primarily focused on comparing cancer patients with and without RA and did not consider cancer treatments (8). Our study was the first to analyze the prognostic factors, including baseline characteristics and treatment options, of patients with LC and preexisting CTDs.

Our study is limited due to its retrospective nature. Baseline assessments were not fully standardized, and several pivotal prognostic factors, including Eastern Cooperative Oncology Group (ECOG) score and prediagnostic weight loss, were not routinely collected. Additionally, 12 patients were lost to follow-up, which reduced the sample size and influenced the quality of the cohort. Furthermore, the number of variables used in the multivariate analysis was relatively overweighed, considering the sample size. Lastly, patients were included in this study over 17 years. Surgical options, chemotherapeutic regimens, and targeted drug treatments for cancer developed during this time, which caused a certain degree of bias. These limitations should be 


\section{Page 10 of 11}

overcome in prospective studies on a larger scale.

\section{Conclusions}

Our study analyzed the clinical characteristics, prognostic factors, and survival of LC patients with preexisting CTDs. LC patients with IIM and SS were shown to have a reduced survival rate; active treatment strategies are required for these patients. Further studies are necessary to confirm our findings.

\section{Acknowledgments}

Funding: This work was supported by grants from the National Natural Science Fund (No. 81801633), Chinese Academy of Medical Sciences Young Medical Talent Award Fund (No. 2018RC320005), Beijing Natural Science Foundation (No. 7182132), Major projects of the Beijing Municipal Science and Technology Commission (No. Z171100002017013) and Capital Special Project for Featured Clinical Application (No. Z151100004015157).

\section{Footnote}

Reporting Checklist: The authors have completed the Strengthening the Reporting of Observational Studies in Epidemiology (STROBE) checklist. Available at http:// dx.doi.org/10.21037/atm-20-1072

Data Sharing Statement: Available at http://dx.doi. org/10.21037/atm-20-1072

Conflicts of Interest: All authors have completed the ICMJE uniform disclosure form (available at http://dx.doi. org/10.21037/atm-20-1072). The authors have no conflicts of interest to declare.

Ethical Statement: The authors are accountable for all aspects of the work in ensuring that questions related to the accuracy or integrity of any part of the work are appropriately investigated and re-solved. This study was conducted in accordance with the Declaration of Helsinki (as revised in 2013) and approved by the Medical Ethics Committee of PUMCH and complied with all ethics committee requirements (protocol ID: S-K1019). Written informed consents have been obtained from all participants.

Open Access Statement: This is an Open Access article distributed in accordance with the Creative Commons Attribution-NonCommercial-NoDerivs 4.0 International License (CC BY-NC-ND 4.0), which permits the noncommercial replication and distribution of the article with the strict proviso that no changes or edits are made and the original work is properly cited (including links to both the formal publication through the relevant DOI and the license). See: https://creativecommons.org/licenses/by-nc-nd/4.0/.

\section{References}

1. Siegel RL, Miller KD, Jemal A. Cancer statistics, 2020. CA Cancer J Clin 2020;70:7-30.

2. Khan SA, Pruitt SL, Xuan L, et al. How does autoimmune disease impact treatment and outcomes among patients with lung cancer? A national SEER-Medicare analysis. Lung Cancer 2018;115:97-102.

3. Khan SA, Pruitt SL, Xuan L, et al. Prevalence of Autoimmune Disease Among Patients With Lung Cancer: Implications for Immunotherapy Treatment Options. JAMA Oncol 2016;2:1507-8.

4. Bernatsky S, Ramsey-Goldman R, Labrecque J, et al. Cancer risk in systemic lupus: an updated international multi-centre cohort study. J Autoimmun 2013;42:130-5.

5. Hemminki K, Liu X, Ji J, Sundquist J, Sundquist K. Effect of autoimmune diseases on risk and survival in histologyspecific lung cancer. Eur Respir J 2012;40:1489-95.

6. Maddison P. Cancer types in dermatomyositis and polymyositis. Lancet 2001;357:1443.

7. Park JK, Yang JA, Ahn EY, et al. Survival rates of cancer patients with and without rheumatic disease: a retrospective cohort analysis. BMC Cancer 2016;16:381.

8. Nayak P, Luo RL, Elting L, et al. Impact of Rheumatoid Arthritis on the Mortality of Elderly Patients Who Develop Cancer: A Population-Based Study. Arthritis Care Res (Hoboken) 2017;69:75-83.

9. Arnett FC, Edworthy SM, Bloch DA, et al. The AmericanRheumatism-Association 1987 Revised Criteria for the Classification of Rheumatoid-Arthritis. Arthritis Rheum 1988;31:315-24.

10. Hochberg MC. Updating the American College of Rheumatology revised criteria for the classification of systemic lupus erythematosus. Arthritis Rheum 1997;40:1725.

11. Vitali C, Bombardieri S, Jonsson R, et al. Classification criteria for Sjogren's syndrome: a revised version of the European criteria proposed by the American-European Consensus Group. Ann Rheum Dis 2002;61:554-8. 
12. Preliminary criteria for the classification of systemic sclerosis (scleroderma). Subcommittee for scleroderma criteria of the American Rheumatism Association Diagnostic and Therapeutic Criteria Committee. Arthritis Rheum 1980;23:581-90.

13. Bohan A, Peter JB. Polymyositis and dermatomyositis (second of two parts). N Engl J Med 1975;292:403-7.

14. Limaye V, Luke C, Tucker G, et al. The incidence and associations of malignancy in a large cohort of patients with biopsy-determined idiopathic inflammatory myositis. Rheumatol Int 2013;33:965-71.

15. So MW, Koo BS, Kim YG, et al. Idiopathic inflammatory myopathy associated with malignancy: a retrospective cohort of 151 Korean patients with dermatomyositis and polymyositis. J Rheumatol 2011;38:2432-5.

16. Wang J, Guo G, Chen G, et al. Meta-analysis of the association of dermatomyositis and polymyositis with cancer. Br J Dermatol 2013;169:838-47.

17. Lu X, Yang H, Shu X, et al. Factors predicting malignancy in patients with polymyositis and dermatomyostis: a systematic review and meta-analysis. PLoS One 2014;9:e94128.

18. Yang Z, Lin F, Qin B, et al. Polymyositis/dermatomyositis and malignancy risk: a metaanalysis study. J Rheumatol 2015;42:282-91.

19. Chow WH, Gridley G, Mellemkjaer L, et al. Cancer risk following polymyositis and dermatomyositis: a nationwide cohort study in Denmark. Cancer Causes Control 1995;6:9-13.

20. Simard JF, Ekberg S, Johansson ALV, et al. What is the impact of chronic systemic inflammation such as rheumatoid arthritis on mortality following cancer? Ann Rheum Dis 2016;75:862-6.

Cite this article as: Yang H, Yao Z, Zhou X, Bing Z, Cao L, Cao Z, Li S, Zhang X, Zhao Y, Zeng X, Zhang F, Liang N. Survival and prognostic factors of lung cancer patients with preexisting connective tissue disease: a retrospective cohort study. Ann Transl Med 2020;8(21):1415. doi: 10.21037/atm-201072
21. Mammen AL, Casciola-Rosen LA, Hall JC, et al. Expression of the dermatomyositis autoantigen $\mathrm{Mi}-2$ in regenerating muscle. Arthritis Rheum 2009;60:3784-93.

22. Levine SM. Cancer and myositis: new insights into an old association. Curr Opin Rheumatol 2006;18:620-4.

23. Kasuya A, Hamaguchi Y, Fujimoto M, et al. TIF1gammaoverexpressing, highly progressive endometrial carcinoma in a patient with dermato-myositis positive for malignancyassociated anti-p155/140 autoantibody. Acta Derm Venereol 2013;93:715-6.

24. Joseph CG, Darrah E, Shah AA, et al. Association of the autoimmune disease scleroderma with an immunologic response to cancer. Science 2014;343:152-7.

25. Adzić TN, Pesut DP, Nagorni-Obradovic LM, et al. Clinical features of lung cancer in patients with connective tissue diseases: a 10-year hospital based study. Respir Med 2008;102:620-4.

26. András C, Bodoki L, Nagy-Vincze M, et al. Retrospective Analysis of Cancer-Associated Myositis Patients over the Past 3 Decades in a Hungarian Myositis Cohort. Pathol Oncol Res 2020;26:1749-55.

27. Enomoto Y, Inui N, Yoshimura K, et al. Lung cancer development in patients with connective tissue diseaserelated interstitial lung disease: A retrospective observational study. Medicine 2016;95:e5716.

28. Saijo A, Hanibuchi M, Goto H, et al. An analysis of the clinical features of lung cancer in patients with connective tissue diseases. Respir Investig 2017;55:153-60.

29. Zhang L, Zhao Q, Yuan F, et al. Lung cancer in patients with and without rheumatoid arthritis: A propensity scorematched survival analysis cohort study. Thorac Cancer 2020;11:1406-13. 